\title{
Erratum to: World Health Organization Guidelines for Feeding Low Birth Weight Infants: Effects of Implementation in First Referral Level Health Facilities in India
}

\author{
Investigators of WHO LBW Feeding Study Group, Delhi, India
}

Ramesh Agarwal ${ }^{1}$

Published online: 6 April 2016

(C) Dr. K C Chaudhuri Foundation 2016

\section{Erratum to: Indian J Pediatr}

DOI 10.1007/s12098-015-1874-4

There has been an inadvertent omission of the list of investigators in the article entitled "World Health Organization Guidelines for Feeding Low Birth Weight Infants: Effects of Implementation in First Referral Level Health Facilities in India" in the online version, which is being appended below:

Investigators of WHO LBW Feeding Study

\section{Kasturba Hospital, New Delhi}

Virmani D (Site PI), Bhatia S, Govil A, Mehta S

\section{Swami Dayanand Hospital, New Delhi}

Jaipal ML (Site PI), Agarwal A, Devgan V, Singh PP, Bisht SS, Grover R

\section{All India Institute of Medical Sciences, New Delhi (Nodal Center) \\ Clinical: Paul VK (PI), Deorari AK, Agarwal R, Mishra S, Thukral A, Chawla D, Sankar MJ, Gupta S, Saksena M, Jain A, Dalal SS, Narayan S, Sivanandan S, George G, Joshi M, Paul JS, Simak K, Sarasan S, Honeymol KC, Lal S}

The online version of the original article can be found at http//dx.doi.org/ 10.1007/s12098-015-1874-4.

\section{Ramesh Agarwal}

ra.aiims@gmail.com

Newborn Health Knowledge Centre (NHKC), Department of Pediatrics, All India Institute of Medical Sciences, Ansari Nagar, New Delhi 110029, India
Biostatistics: Sreenivas V, Suresh SS, Yadav CP

Protocol and study tools development : Agarwal R, Paul VK, Deorari AK, Chawla D, Sankar MJ, Thukral A, Lal S, Sreenivas V

Study implementation: Agarwal R, Mishra S, Thukral A, Sankar MJ, Chawla D, Jain A, Bisht SS, Maria A, Dalal SS, Narayan S, Jain A, Sivanandan S, George G, Joshi M, Jessy, Simak K, Sarasan S, Honeymol KC

Quality assurance: Agarwal R, Paul VK, Chawla D, Sankar MJ Data management: Lal S, Suresh SS, Agarwal R, Sankar MJ Data analysis: Gupta S, Yadav CP, Sankar MJ, Agarwal R, Suresh SS

Manuscript writing: Gupta S, Agarwal R, Sankar MJ, Paul VK 